\title{
Nefropatía por tóxicos: la cocaína y su potente daño renal
}

\author{
Toxic nephropathy: cocaine and its potent kidney damage
}

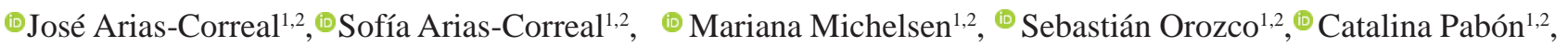

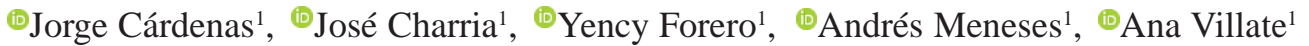 \\ 'Departamento Médico, Clínica Palermo, Bogotá D.C., Colombia. \\ ${ }^{2}$ Facultad de Medicina, Universidad de La Sabana, Chía, Colombia.
}

\begin{abstract}
Resumen
El consumo de sustancias ilícitas en menores de 16 años y en adultos jóvenes se incrementa cada día en Colombia, por lo cual se presentan complicaciones asociadas que sería inusual encontrar en este grupo poblacional. El presente reporte de caso muestra cómo el uso de cocaína llevó a un paciente joven a desarrollar daño renal agudo con requerimiento de terapia de reemplazo renal, lo que en los hallazgos histológicos puede corresponder a una glomerulonefritis rápidamente progresiva o a una enfermedad tubulointersticial tipo necrosis tubular aguda o nefritis intersticial aguda.

Palabras clave: drogas ilícitas, insuficiencia renal, necrosis tubular aguda (DeCS).

doi:http://dx.doi.org/10.22265/acnef.7.2.390

Abstract

In Colombia, the consumption of illicit substances increases daily. The increase and related consumption in the population involves both young people under 16 and young adults. Therefore, there are complications associated with the consumption of these substances that otherwise, would be unusual to find in this population group. In this case report, we will review how the use of cocaine led a young patient to the development acute kidney injury requiring renal replacement therapy, which in the histological findings may correspond to rapidly progressive glomerulonephritis, or tubulointerstitial disease either acute renal tubular necrosis or acute interstitial nephritis.
\end{abstract}

Keywords: street drugs, renal insufficiency, acute kidney tubular necrosis (MeSH).

doi:http://dx.doi.org/10.22265/acnef.7.2.390

\section{Introducción}

$\mathrm{E}$ n Colombia, la situación referente al consumo de drogas lícitas como el alcohol o el tabaco en la adolescencia y la adultez temprana ( $<40$ años) es alarmante: según el Observatorio de Drogas de Colombia ${ }^{1}$, para el año 2017 alrededor del $86,3 \%$ de los jóvenes menores de 16 años ya habían consumido alcohol al menos una vez en la vida.

Por otra parte, para el 2016, hasta el $16 \%$ de los escolares en secundaria declaró haber consumido alguna vez en la vida una sustancia ilícita como marihuana, cocaína, bazuco, éxtasis, popper y otros alucinógenos populares en este grupo etario. Se ha planteado que la exposición temprana a estas sustancias puede desencadenar fácilmente una adicción a las mismas². Por consiguiente, es relevante comprender el fenómeno social del que dicha exposición y consumo se deriva, así como entender que, aunado a la presencia de las alteraciones de índole sociocultural, también se pueden presentar alteraciones de tipo orgánico que afectan el estado de salud de los sujetos consumidores, lo que se convierte entonces en un problema de salud pública.

En la literatura se han descrito múltiples mecanismos mediante los cuales se puede desarrollar una lesión renal aguda debido al consumo de sustancias ilícitas como la cocaína, tales como alteración a nivel glomerular y en el componente túbulo-intersticial. Con relación a este último, los dos patrones de presentación más relevantes y frecuentes son la necrosis tubular aguda (NTA) y la nefritis intersticial aguda (NIA) ${ }^{3}$.

Dentro de la aproximación inicial a los pacientes de edad temprana con daño renal agudo, la anamnesis es esencial para elaborar una buena historia clínica, la cual debe ser complementada con un examen físico

Citación: Arias-Correal J, Arias-Correal S, Michelsen M, Orozco S, Pabón C, Cárdenas J, et al. Nefropatía por tóxicos: la cocaína y su potente daño renal. Rev. Colomb. Nefrol. 2020;7(2):98-103. https://doi.org/10.22265/acnef.7.2.390

Recibido: 24.02.20, Aceptado: 19.05.20, Publicado en línea: 05.08.20

Correspondencia: José Arias-Correal, joseariascorreal@gmail.com 
exhaustivo en el que se tenga en cuenta el patrón de daño renal que presenta el paciente. Estas son herramientas útiles para esclarecer si la persona consume o no sustancias ilícitas, como en esta ocasión la cocaína.

En este sentido, tal como se evidencia en el presente reporte de caso, la exposición a drogas ilícitas es un factor que se debe tener en consideración al momento de tratar alteraciones de la función renal y daño renal agudo en pacientes jóvenes.

\section{Presentación del caso}

Paciente masculino de 19 años que presentó como antecedente de importancia consumo de alcohol (tres veces por semana hasta la embriaguez) y tabaquismo (12 cigarrillos aproximadamente al día desde hace 6 años), y quien adicionalmente indicó consumo de marihuana una o dos veces al día, cocaína cada semana y otras drogas que no especificóa. El joven no indicó diagnóstico de alguna patología previa u otro antecedente de importancia, pero se logró documentar la presencia de factores de riesgo para desarrollo de enfermedades de transmisión sexual, pues, según refirió, era bisexual, promiscuo y no utilizaba medidas de protección como preservativo.

El paciente ingresó a la institución por cuadro clínico de 6 horas de evolución consistente en dolor abdominal severo, localizado en hipocondrio izquierdo, irradiado a región dorso-lumbar izquierda y asociado a episodios eméticos y hematuria macroscópica, además de sintomatología irritativa urinaria dada por disuria, urgencia miccional y tenesmo vesical. Durante su estancia en urgencias se descartó patología quirúrgica abdominal y dentro del proceso de exploración diagnostica se realizaron estudios paraclínicos iniciales, con los que se pudo descartar compromiso tanto anatómico como infeccioso (Tabla 1).

Se documentó daño renal agudo que fue clasificado mediante la escala de severidad AKI-KDIGO en estadio 3 dada la marcada elevación de los niveles de creatinina. Se realizó relación BUN/Creatinina con resultado $<20$, por lo que se consideró que una patología intrínseca renal era la causante del daño renal. Debido a que se trataba de un paciente joven, se tomó biopsia renal con la que se evidenció la presencia de compromiso tubular dado por necrosis tubular aguda; se sospechó que esta última era secundaria a sus antecedentes de consumo de sustancias tóxicas. Dada la alteración marcada de la función renal con cálculo de tasa de filtrado glomerular por medio de CKD-EPI con estadio V $\left(<15 \mathrm{~mL} / \mathrm{min} / 1.73 \mathrm{~m}^{2}\right)$, se decidió instaurar soporte con terapia de remplazo renal mediante hemodiálisis con ciclos de cuatro horas al día por seis días, a partir de lo cual se logró una recuperación progresiva de la función renal (Figura 1).

El paciente tuvo una evolución satisfactoria de los síntomas y retornó al gasto urinario previo, por

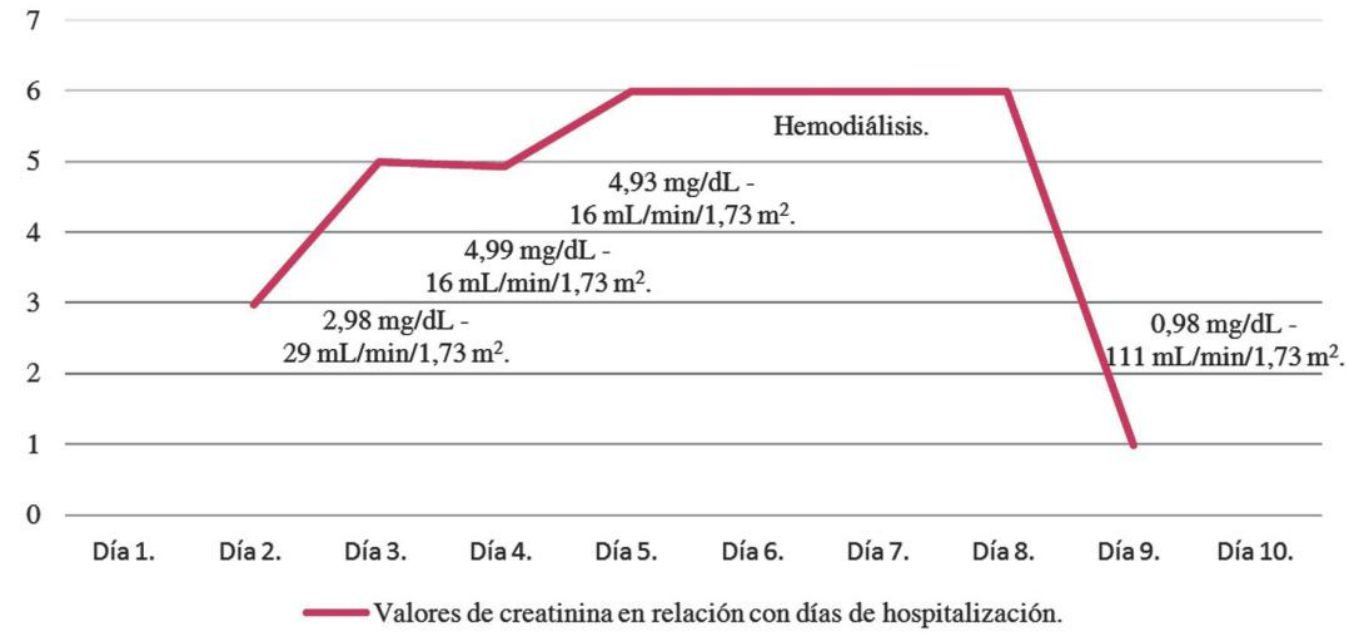

Figura 1. Valores de creatinina y tasa de filtrado glomerular por CKD-EPI, en relación a los días de hospitalización. Fuente: elaboración propia. 
Tabla 1. Resultados de laboratorio.

\begin{tabular}{|c|c|c|c|}
\hline \multicolumn{2}{|r|}{ Laboratorio } & \multirow{2}{*}{$\begin{array}{c}\text { Resultado } \\
26,1 \mathrm{mg} / \mathrm{dL}\end{array}$} & \multirow{2}{*}{$\begin{array}{c}\begin{array}{c}\text { Valores de } \\
\text { referencia }\end{array} \\
0-20\end{array}$} \\
\hline \multirow{9}{*}{$\begin{array}{l}\text { Química } \\
\text { sanguínea }\end{array}$} & Nitrógeno ureico sanguíneo & & \\
\hline & Creatinina en suero & $2,98 \mathrm{mg} / \mathrm{dL}$ & $0,5-1,2$ \\
\hline & Bilirrubina total & $0,27 \mathrm{mg} / \mathrm{dL}$ & $0-1,5$ \\
\hline & Bilirrubina directa & $0,12 \mathrm{mg} / \mathrm{dL}$ & $0-1$ \\
\hline & Bilirrubina indirecta & $0,15 \mathrm{mg} / \mathrm{dL}$ & $0-1$ \\
\hline & Aspartato aminotransferasa & $23 \mathrm{U} / \mathrm{L}$ & $5-39$ \\
\hline & Alanina aminotransferasa & $13 \mathrm{U} / \mathrm{L}$ & $5-40$ \\
\hline & Sodio & $138 \mathrm{mmol} / \mathrm{L}$ & $136-145$ \\
\hline & Potasio & $5,12 \mathrm{mmol} / \mathrm{L}$ & $3,5-5,3$ \\
\hline \multirow{8}{*}{$\begin{array}{l}\text { Biometría } \\
\text { hemática }\end{array}$} & Leucocitos & 12.630 & $4.000-10.000$ \\
\hline & Neutrófilos & 8.900 & - \\
\hline & Linfocitos & 1.520 & - \\
\hline & Monocitos & 1.530 & - \\
\hline & Otros & 680 & - \\
\hline & Hematocrito & $38,8 \%$ & $40-55$ \\
\hline & Hemoglobina & $13,5 \mathrm{~g} / \mathrm{dL}$ & $12-18$ \\
\hline & Plaquetas & 400.000 & $150.000-450.000$ \\
\hline \multirow{5}{*}{$\begin{array}{l}\text { Inmunología } \\
\text { infecciosa }\end{array}$} & Virus de VIH 1 y 2. ELISA 4ta generación & $0,16 \mathrm{U} / \mathrm{L}$ & $<0,99$ \\
\hline & Hepatitis B antígeno de superficie & $0,32 \mathrm{U} / \mathrm{L}$ & $<0,99$ \\
\hline & Hepatitis B anti-CORE total & $2.210 \mathrm{U} / \mathrm{L}$ & $>1$ \\
\hline & Hepatitis $\mathrm{C}$ anticuerpos & $0,15 \mathrm{U} / \mathrm{L}$ & $<0,99$. \\
\hline & Prueba no treponémica, RPR & No reactiva & No reactiva \\
\hline \multirow{13}{*}{$\begin{array}{l}\text { Análisis } \\
\text { urinario }\end{array}$} & Color & Amarillo & - \\
\hline & Aspecto & Lig. Turbio & - \\
\hline & Densidad & 1,02 & - \\
\hline & Estearasa leucocitaria & Negativo & - \\
\hline & Nitritos & Negativo & - \\
\hline & Proteinas & $75 \mathrm{mg} / \mathrm{dL}$ & - \\
\hline & Glucosa & Normal & - \\
\hline & Cetonas & Negativo & - \\
\hline & Eritrocitos & $250 / \mathrm{uL}$ & - \\
\hline & Bacterias & + & - \\
\hline & Células epiteliales & $1 / \mathrm{uL}$ & - \\
\hline & Leucocitos & $15 / \mathrm{uL}$ & - \\
\hline & Hematies & $1 / \mathrm{uL}$ & - \\
\hline
\end{tabular}

Fuente: Elaboración propia.

lo que se le dio el alta hospitalaria sin tratamiento adicional, pero con controles por medicina interna, nefrología y soporte por psiquiatría para manejo integral.

\section{Discusión}

La cocaína es una sustancia derivada de la planta Erythroxylum coca que al actuar como inhibidor de 
recaptación de catecolaminas, (serotonina, norepinefrina y dopamina) sirve como estimulante y tiene la capacidad de generar supresión del apetito e inclusive efectos anestésicos ${ }^{4}$. Esta sustancia se puede consumir de dos formas: soluble como una sal mediante el contacto con mucosas (inhalación por mucosa nasal, aplicación intraocular o intravaginal, e incluso como supositorio anal) o insoluble, conocida también como crack, mediante inyección directa en el tracto sanguíneo 5 .

Según datos epidemiológicos, el consumo de sustancias ilícitas a nivel global está generando dificultades con los recursos de los sistemas de salud enfocados en el control de este problema de salud pública debido a los gastos producto de las sobredosis y las complicaciones que pueden sufrir las personas adictas. Por ejemplo, en Estados Unidos para el año 2011 se reportó un incremento de 5 veces en el número de muertes asociadas a sobredosis en comparación con datos de los años ochenta ${ }^{6}$.

Los opioides no prescritos medicamente y la marihuana son las sustancias ilícitas que más impacto generan en población menor de 12 años, y según lo mencionado por Mansoor, et al. ${ }^{7}$, aproximadamente 54 billones de dólares se utilizan para el soporte de los pacientes que presentan abuso de estas y otras sustancias prohibidas.
La cocaína, así como otros tóxicos (Tabla 2), pueden causar daño renal agudo, además los pacientes que tengan un consumo pesado de esta sustancia pueden desarrollar un amplio espectro de complicaciones mediante diversos mecanismos, desde el componente hemodinámico y sistémico, hasta la inducción de daño por aumento de estrés oxidativo ${ }^{8}$.

El mecanismo de acción de la cocaína permite bloquear la recaptación de norepinefrina, de tal manera que aumenta el potencial simpático y su acción sobre el sistema cardiovascular ${ }^{9}$. De esta forma, en una gran mayoría de pacientes la exposición a dicha sustancia se asocia con la aparición de infartos renales debido a su potente acción vasoconstrictora; generalmente, las manifestaciones clínicas de estos episodios se presentan con dolor severo, náuseas y emesis, por lo que es importante siempre realizar el proceso de diagnóstico adecuado para descartar patologías de índole quirúrgico.

La cocaína, por su potente capacidad de vasoconstricción, a nivel hemodinámico puede generar alteraciones adicionales como disminución del flujo arterial renal e isquemia sin infarto, compromiso que depende del tiempo de consumo y de la presencia de comorbilidades que en cada caso faciliten la instauración de un daño permanente sin capacidad de reversibilidad de la obstrucción del flujo. En relación

Tabla 2. Daño renal por sustancias tóxicas.

\begin{tabular}{|l|l|l|}
\hline \multicolumn{2}{|c|}{ Sustancia. } & \multicolumn{1}{c|}{ Daño túbulo intersticial. } \\
\hline $\begin{array}{l}\text { Cannabinoides } \\
\text { sintéticos }\end{array}$ & "Spice" "K2" & NTA \\
\cline { 2 - 3 } $\begin{array}{l}\text { Catinonas } \\
\text { sintéticas }\end{array}$ & "Scienso herbal & NIA \\
\hline MDMA & "Éxtasis" & NTA \\
\hline $\begin{array}{l}\text { Anabólicos } \\
\text { androgénicos }\end{array}$ & Esteroides y suplementos nutricionales. & NTA - Nefropatía por sales biliares \\
\hline Solventes inhalados & \\
\hline \multirow{2}{*}{ Heroína } & ATR \\
\cline { 3 - 3 } & Síndrome de Fanconi \\
\hline Cocaína & NTA \\
\cline { 2 - 3 } & Nefropatía por cristales de heroína \\
\hline
\end{tabular}

NTA: Necrosis tubular aguda; NIA: nefritis intersticial aguda; ATR: acidosis tubular renal. Fuente: elaboración con base en Nanavati \& Herlitz ${ }^{8}$. 
con el sistema tubular, los metabolitos de la cocaína producen vasoconstricción directa en el lecho renal y alteran la mitocondria por depleción de glutatión ${ }^{10}$; ante la pérdida de los mecanismos encargados de limitar el daño por estrés oxidativo y el compromiso muscular que conlleva a rabdomiólisis, se genera apoptosis y por ende NTA o insuficiencia renal agu$\mathrm{da}^{11}$. Adicionalmente, la cocaína tiene el potencial de inducir la activación del sistema renina-angiotensinaaldosterona de manera directa $\mathrm{y}$, en consecuencia, generar fibrosis a nivel mesangial ${ }^{12}$.

De esta forma, el resultado final de un consumo crónico de cocaína es un proceso vasculítico relacionado con anticuerpos anticitoplasma de neutrófilos (ANCA) en el que se determina como pilar terapéutico el detener el consumo de este tóxico de manera inmediata, controlar la tensión arterial y realizar un manejo enfocado en prevenir el uso de nefrotóxicos, o dejarlos como última instancia, dependiendo de la severidad del cuadro clínico. El uso de inmunomodulares como los corticoesteroides están indicados a dosis de $1 \mathrm{mg} / \mathrm{kg}$ y la terapia de remplazo renal con hemodiálisis se puede implementar, tal como se hizo en el caso presentado ${ }^{13}$.

\section{Conclusión}

Al ser Colombia un país con alta prevalencia de consumo de sustancias ilícitas, incluso desde edades tempranas ( $<16$ años), el diagnóstico diferencial en pacientes consumidores siempre debe estar presente, además se debe tener en cuenta que el consumo de cocaína tiene un espectro múltiple de daño a nivel renal e incluso sistémico. En consecuencia, el médico que realice el proceso de aproximación diagnostica debe tener en cuenta la presentación de daño renal agudo en un contexto de consumidor de cocaína, el tiempo de exposición a esta sustancia, la adopción de medidas enfocadas en el cese del consumo y el acompañamiento continuo al paciente, los cuales deben propenderse para no solo preservar la función renal, sino para prevenir las complicaciones, incluida la muerte. De igual forma, es importante que, en caso de requerirse, se considere como una opción terapéutica la instauración temprana de terapias de remplazo renal como la hemodiálisis para evitar el desarrollo de enfermedad renal crónica.

\section{Agradecimientos}

A la paciencia de nuestras familias por darnos el apoyo más preciado en razón del tiempo utilizado para la preparación de este manuscrito y la atención que presentamos a nuestros pacientes.

\section{Conflicto de intereses y financiación}

Ninguno declarado por los autores.

\section{Contribuciones de los autores}

Por parte del grupo de departamento médico, se realizó el manuscrito y búsqueda de la información, José Arias-Correal y Sofía Arias-Correal lideran el proyecto, con ayuda de personal asistencial José Charria, Jorge Cárdenas, Yency Forero, Ana Villate y Andrés Meneses quienes complementan la discusión del manuscrito; se complementa búsqueda de la información con literatura reciente y agregando comentarios por parte de Mariana Michelsen, Sebastián Orozco y Catalina Pabón, quienes también resumen el caso clínico, como también obtienen la información por parte del paciente. Por último, los que lideran el manuscrito, realizan la corrección con parte del personal asistencial del departamento médico y el Internista a cargo José Charria, avala el sometimiento del mismo a la Revista Colombiana de Nefrología.

\section{Responsabilidades éticas}

Los autores declaran que para esta investigación no se realizaron experimentos en seres humanos ni en animales.

\section{Derecho a la privacidad y consentimiento informado}

Los autores declaran que en este artículo no aparecen datos de pacientes. 


\section{Referencias}

1. Colombia. Observatorio de Drogas de Colombia (ODC). Estudio de consumo de sustancias psicoactivas en el Sistema de Responsabilidad Penal para Adolescentes ( SRPA ) Colombia Resumen ejecutivo. Bogotá D.C.: ODC; 2018 [citado sep 20 2020]. Disponible en:

ht t p : / / w w w odc.gov.co/Portals/1/publicaciones/pdf/consumo/estudios/nacionales/ CO3142018_estudio_consumo_sustancias_psicoactivas_SRPA_2018.pdf.

2. Colombia. Observatorio de Drogas de Colombia (ODC). Estudio nacional de consumo de sustancias psicoactivas en población escolar colombiana - 2016 [Internet]. Bogotá D.C.: (ODC); 2016[citado sep 20 2020]. Disponible en: http://www.odc.gov.co/Portals/1/publicaciones/pdf/consumo/estudios/nacionales/CO03142016_estudio_consumo_escolares_2016.pdf.

3. Pendergraft WF 3rd, Herlitz LC, Thornley-Brown D, Rosner M, Niles JL. Nephrotoxic Effects of Common and Emerging Drugs of Abuse. Clin J Am Soc Nephrol. 2014; 9(11):1996-2005 . https://dx.doi.org/10.2215/CJN.00360114.

4. EE. UU. National INstitute on Drug Abuse. Cocaine Research Report. What is Cocaine?. Bethesda, MD: National Institutes of Health; 2016 [citado sep 29 2020]. Disponible en: https://www.drugabuse.gov/publications/research-reports/cocaine/what-cocaine.

5. Pomara C, Cassano T, Errico SD, Bello S, Romano AD, Riezzo I, et al. Data Available on the Extent of Cocaine Use and Dependence?: Biochemistry, Phar- macologic Effects and Global Burden of Disease of Cocaine Abusers. Curr Med Chem. 2012;19(53):5647-57. https://dx.doi.org/10.2174/092986712803988811.

6. Warner M, Chen LH, Makuc DM, Anderson RN, Miniño AM. Drug poisoning deaths in the United States, 1980-2008. NCHS data brief. 2011;81(81):1-8. https://dx.doi.org/10.1002/9780470976012.ch1

7. Mansoor K, Kheetan M, Shahnawaz S, Shapiro AP, Patton-Tackett E, Dial L, et al. Systematic Review of nephrotoxicity of drugs abuse, 2005 - 2016. BMC Nephrology. 2017; 18:379-394.

8. Nanavati A, Herlitz LC. Tubulointerstitial Injury and Drugs of Abuse. Adv Chronic Kidney Dis. 2017;24(2):80-5. http://dx.doi.org/10.1053/j.ackd.2016.09.008.

9. Vongpatanasin W, Mansour Y, Chavoshan B, Arbique D, Victor RG. Cocaine Stimulates the Human Cardiovascular System via a Central Mechanism of Action. Circulation. 1999;100(5):497-502. http://dx.doi.org/10.1161/01.cir.100.5.497.

10. Valente MJ, Henrique R, Vilas-Boas V, Silva R, Bastos Mde L, Carvalho F, et al. Cocaine-induced kidney toxicity?: an in vitro study using primary cultured human proximal tubular epithelial cells. Arch Toxicol. 2012;86(2):249-61.

http://dx.doi.org/10.1007/s00204-011-0749-3.

11. Valente MJ, Carvalho F, Bastos ML, de Pinho PG, Carvalho M. Contribution of Oxidative Metabolism to Cocaine-Induced Liver and Kidney Damage. Curr Med Chem.. 2012;19(33):5601-6. http://dx.doi.org/10.2174/092986712803988938.

12. Trouve R, Latour C, Nahas G. Cocaine and the renin-angiotensin system. Adv Biosci. 1991;80:165-76.

13. Mcgrath MM, Isakova T, Rennke HG, Mottola AM, Laliberte KA, Niles JL. Contaminated Cocaine and Antineutrophil Cytoplasmic Antibody-Associated Disease. Clin J Am Soc Nephrol. 2011;6(12):2799-805. http://dx.doi.org/10.2215/CJN.03440411. 